\title{
Efectos bioquímicos y clínicos del hialuronato oral en caballos Andaluces jóvenes con osteocondrosis tarsocrural ${ }^{\#}$
}

\author{
Biochemical and clinical effects of oral hyaluronan in young Andalucian \\ horses with tarsocrural osteochondrosis \\ JU Carmona ${ }^{a^{*}}$, D Argüelles ${ }^{b}$, M Prades ${ }^{\mathrm{c}}$ \\ ${ }^{a}$ Grupo de Investigación Terapia Regenerativa, Departamento de Salud Animal, Universidad de Caldas, Calle 65 № 26-10, \\ Manizales, Caldas, Colombia.

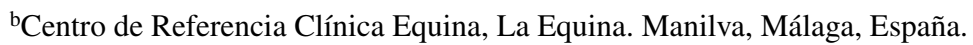 \\ 'Departamento de Medicina y Cirugía Animal, Universidad Autónoma de Barcelona, \\ Edificio V. 08193, Bellaterra, Barcelona, España.
}

\begin{abstract}
SUMMARY
The aim of this study was to evaluate the clinical and biochemical effects of an oral preparation of hyaluronic acid (HA) (Hyal-Joint ${ }^{\circledR}$ ) in young Andalucian horses with osteochondrosis (OCD) of the tarsocrural joint. Our hypotheses were that the administration of oral HA is safe, diminishes the degree of synovial effusion and the synovial levels of nitric oxide (NO) and prostaglandin E2 (PGE2) and increases the plasmatic and synovial levels of HA. Eleven Andalucian young horses with OCD of the tarsocrural joint were included in a double blinded controlled study. Six horses received oral HA at $250 \mathrm{mg}$ /day, during 60 days (T60) and 5 horses received a placebo. The initial values from the degree of synovial effusion, NO, PGE2 and HA synovial levels (affected joints) and HA plasmatic levels were obtained. A cytological analysis with determination of its total protein levels was performed. The horses were evaluated for same parameters at the end of the treatment (T60) and 30 days later (T90). Statistically significant differences for each one of the parameters evaluated in both groups were not observed through time (beginning, T60 and T90). Nevertheless, the horses treated with oral HA presented tendencies to show a minor degree of synovial effusion and higher synovial HA levels. Oral HA did not produce any adverse clinical effect in the horses treated during 60 days, and it is safe and well tolerated in horses at a dose of $250 \mathrm{mg} /$ day.
\end{abstract}

Palabras clave: equino, osteocondrosis, hialuronato oral, óxido nítrico, prostaglandina E2.

Key words: equine, osteochondrosis, oral hyaluronan, nitric oxide, prostaglandin E2.

\section{INTRODUCCIÓN}

La osteocondrosis (OCD) es una enfermedad multifactorial con un componente hereditario, catalogada dentro del complejo de enfermedades ortopédicas del crecimiento en el caballo (Wittwer y col 2006). Se caracteriza por un fallo en la osificación endocondral del complejo epifisiario-articular. Las articulaciones equinas más afectadas son la tarsocrural, femoropatelar y metacarpofalangiana (McIlwraith 1996 ${ }^{\mathrm{a}}$ ). La incidencia de la enfermedad en Europa puede llegar a ser hasta de un $30 \%$ (Wittwer y col 2006). La OCD produce cuantiosas pérdidas económicas a la industria equina, representadas por la devaluación del potencial atlético y reproductivo de los ejemplares afectados (Robert y col 2006).

Clínicamente, las articulaciones afectadas por la enfermedad pueden manifestar efusión sinovial, con diferentes grados de distensión articular. En muchas ocasiones no

Aceptado: 18.03.2009.

* Financiado por Bioibérica S.A., Barcelona, España.

\# Departamento de Salud Animal, Universidad de Caldas, Calle 65 № 26-10, Manizales, Caldas, Colombia; carmona@ucaldas.edu.co. se observa cojera; sin embargo, este signo puede aparecer cuando los caballos jóvenes inician su entrenamiento (McIlwraith 1996'a). Esta situación es cierta, sobre todo cuando las lesiones osteocondrales están ubicadas en sitios de carga articular y gran movilidad (e.j.: cresta intermedia de la tibia) (de Grauw y col 2006, van Weeren 2006).

Una articulación con OCD puede tener fragmentos osteocondrales libres, exposición focalizada de hueso subcondral y sinovitis (McIlwraith 1996'a). Este cuadro patológico presenta alteraciones bioquímicas del ambiente articular, las cuales perpetúan la sinovitis con la consecuente destrucción del cartílago articular sano (de Grauw y col 2006, Gangl y col 2007). Numerosos agentes proinflamatorios primarios, tales como la interleucina 1 (IL-1), activan la expresión de enzimas inflamatorias, como la ciclooxigenasa 2 (COX-2), lipooxigenasa (LPX), sintetasa inducible de óxido nítrico (iNOS) y metaloproteinasas de matriz (MMPs), entre otras (Palmer y Bertone 1994, Carmona y Giraldo-Murillo 2007). Estas enzimas a su vez producen numerosos metabolitos inflamatorios secundarios (ej.: prostaglandina E2 (PGE2), leucotrieno B4 (LTB4) y óxido nítrico (NO)) que pueden desencadenar osteoartritis (OA) (McIlwraith 1996 ${ }^{\text {b }}$, Carmona y Giraldo-Murillo 2007). 
Anualmente son gastados miles de dólares en el tratamiento de caballos con OCD. Muchos propietarios siguen el consejo de sus veterinarios y optan de manera temprana por un tratamiento quirúrgico (artroscopia) para sus caballos (McIlwraith 1996a, van Weeren 2006). La artroscopia acompañada de la remoción de fragmentos osteocondrales, desbridamiento de la superficie articular afectada y lavado articular puede ser considerada como el tratamiento más apropiado de la enfermedad (McIlwraith $\left.1996^{a}\right)$. La intervención artroscópica temprana puede, en la mayoría de las veces, frenar la evolución de la patología y evitar su progresión hacia una enfermedad articular degenerativa (osteoartritis) (Carmona y Giraldo-Murillo 2007). Sin embargo, en muchas ocasiones y a pesar de la cirugía algunos pacientes continúan con efusión sinovial (McIlwraith $1996^{\mathrm{b}}$, van Weeren 2006), que a la larga puede terminar en OA. Es necesario aclarar, también, que debido al alto costo de los procedimientos artroscópicos y a los gastos asociados con dicho procedimiento, muchos caballos con OCD no son operados y algunas veces sus propietarios optan por un tratamiento médico.

Desde el punto de vista biomédico es importante considerar que la OCD es una entidad con un mecanismo fisiopatológico común en las diversas especies de mamíferos (humanos, equinos, caninos y porcinos, entre otros) (McIlwraith 1996a). El caballo con enfermedad (OCD) natural representa un excelente modelo animal para conocer y extrapolar los mecanismos patogénicos de la alteración a los seres humanos y otras especies animales y para conocer la respuesta de la enfermedad frente a diversos tratamientos médicos o quirúrgicos (Olstad y col 2007).

El ácido hialurónico (HA) es un compuesto natural del líquido sinovial. Es un glicosaminoglicano no sulfatado, producido por las células sinoviales (Howard y McIlwraith 1996). Esta sustancia es empleada como tratamiento intravenoso o intraarticular de artropatías equinas. Su farmacocinética ha sido descrita por ambas vías (Popot y col 2004). El primer informe sobre el uso del HA se remonta a la década de los años 70 del siglo pasado. A partir de esa fecha, se han documentado varios estudios in vivo e in vitro, numerosos informes anecdóticos y ensayos clínicos en caballos (Howard y McIlwraith 1996). La principal acción del HA es promover la lubricación articular; sin embargo, su mecanismo de acción no ha sido totalmente entendido. Se ha postulado que el HA podría tener efecto antiartrítico por dos razones: 1) Modula la respuesta biológica mediada por los receptores específicos de la membrana celular de leucocitos y otras células articulares. 2) Interfiere mecánicamente con la interacción de varias proteínas catabólicas y sus receptores celulares específicos (Howard y McIlwraith 1996, Frean y col 1999, Tung y col 2002a).

Se ha demostrado que el HA disminuye la producción in vitro de PGE2 en sinoviocitos humanos (Howard y McIlwraith 1996, Frean y col 1999), pero no en condrocitos equinos condicionados con IL-1 (Tung y col 2002 $2^{\mathrm{a}, \mathrm{b}}$ ). Por otra parte, en un estudio experimental de fragmentación osteocondral en caballos la administración intravenosa de $40 \mathrm{mg}$ de HA cada 7 días durante tres semanas redujo de manera estadísticamente significativa el grado de cojera, grado histológico de sinovitis y las concentraciones sinoviales de PGE2 a los 72 días postoperatorios en los caballos tratados respecto a los caballos del grupo control (Kawcak y col 1997). La suma de las observaciones reportadas hace pensar que el HA posee efectos bioquímicos diferentes en sistemas in vitro en comparación con modelos animales.

Se ha descrito el uso de una preparación oral de HA (gel) como tratamiento complementario de caballos con OCD del tarso sometidos a artroscopia (Bergin y col 2006). Los resultados obtenidos en ese estudio doble ciego demostraron que los caballos tratados con HA oral mostraron disminución estadísticamente significativa del grado de distensión sinovial postoperatoria, frente a un grupo placebo (Bergin y col 2006). Los resultados de ese trabajo son esperanzadores; sin embargo, en ese estudio no se realizó evaluación bioquímica del líquido sinovial de los animales, por lo que no se pudo conocer el mecanismo de acción que indujo la disminución de la efusión sinovial de los caballos tratados con HA oral (Bergin y col 2006). En la literatura revisada no se halló documentación sobre el comportamiento farmacocinético de preparaciones orales de HA en caballos. Sin embargo, recientemente se describió un porcentaje de absorción aproximado del $13 \%$ de HA radiomarcado en el tracto gastrointestinal de perro y rata (Balogh y col 2008). Ambos animales son monogástricos como el caballo.

En este trabajo se presentan los resultados de un estudio doble ciego en el que se evaluaron los efectos de una preparación comercial de ácido hialurónico oral (Hyal-Joint ${ }^{\circledR}$, Bioibérica S.A, Barcelona, España) en caballos Andaluces jóvenes con OCD del tarso. Los objetivos de este trabajo fueron: (1) evaluar el efecto clínico del HA oral durante un periodo de dos meses mediante análisis del grado de distensión (efusión) sinovial y grado de cojera; (2) determinar el efecto de HA oral sobre las concentraciones sinoviales de NO, PGE2 y HA de las articulaciones comprometidas y (3) medir las concentraciones plasmáticas de HA en los caballos del estudio.

Las hipótesis de este estudio fueron que el grupo de caballos tratados al azar con HA oral deberían presentar una mejor respuesta clínica al tratamiento (disminución del grado de efusión sinovial y de cojera). Paralelamente, las concentraciones sinoviales de NO y PGE2 deberían ser inferiores y las concentraciones plasmáticas y sinoviales de HA deberían ser mayores respecto al grupo control. Adicionalmente, el HA oral no debería producir ninguna reacción adversa en los caballos tratados, tales como fiebre, anorexia o diarrea, entre otras.

\section{MATERIAL Y MÉTODOS}

Este estudio clínico doble ciego controlado, prospectivo, fue aceptado por las directivas del Hospital Clínico 
Veterinario de la Universidad Autónoma de Barcelona, España. El trabajo fue realizado en dos fases: trabajo de campo y análisis de laboratorio. El trabajo de campo fue realizado en varias yeguadas localizadas en las provincias centrales de la comunidad autónoma de Cataluña, España. Los dueños de los caballos firmaron autorización consentida, luego de ser informados sobre la naturaleza experimental del estudio y de las posibles complicaciones derivadas de la punción articular. Los caballos enrolados eran de yeguadas con condiciones ambientales, nutricionales y de manejo similares.

\section{CABALLOS Y CRITERIOS DE INCLUSIÓN EN EL ESTUDIO}

Once caballos de raza Andaluz fueron incluidos, 10 machos y 1 hembra, con un rango de edad entre 8-36 meses. El criterio para incluir un caballo se basó en dos factores: edad (menores de 3 años) y presencia radiográfica (bilateral o unilateral) de fragmentos osteocondrales en la articulación tarsocrural, acompañados de signos clínicos de OCD, tales como efusión sinovial y cojera. Esta última variable no fue considerada estrictamente como un criterio de inclusión, ya que generalmente la OCD no produce cojera evidente (McIlwraith 1996).

\section{EXAMEN CLÍNICO Y DEL APARATO LOCOMOTOR}

Se realizó hemograma y perfil bioquímico general (proteína total, albúmina, AST, creatinina y glucosa) a cada caballo, con el objetivo de incluir animales sin enfermedad sistémica. Seguidamente, se realizó evaluación del aparato locomotor (palpación, valoración pasiva de la flexibilidad articular y valoración dinámica del grado de cojera). Los caballos que presentaban efusión sinovial (uni o bilateral) del tarso, acompañada o no de cojera, fueron sometidos a un estudio radiológico mediante las cuatro proyecciones estándar (Wittwer y col 2006).

Graduación del grado de efusión sinovial. Se realizó evaluación semicualitativa del grado de efusión sinovial, para lo cual se combinaron los resultados de las observaciones clínicas y los hallazgos ecográficos de cada articulación. La valoración clínica incluyó la evaluación del aspecto externo de la(s) articulación(es) afectada(s) en comparación con la articulación contralateral sana o con la articulación de un animal sano de la misma edad. Se evaluaron la temperatura, textura de la cápsula, grado de movimiento articular, grado de dolor durante la palpación y movimiento pasivo articular. El examen ecográfico incluyó: evaluación de la superficie articular, cantidad de líquido sinovial, grosor $\mathrm{y}$ aspecto de la membrana sinovial y sus vellosidades y presencia de fragmentos osteocondrales.

El grado de efusión sinovial (distensión articular) fue calificado en una escala de 0 a 3 , donde $0=$ articulación de aspecto normal. 1 = efusión levemente palpable, sin engrosamiento de la cápsula articular. 2 = efusión palpable, con engrosamiento capsular moderado y una reacción dolorosa leve durante la palpación profunda. 3 = efusión severa, con la membrana sinovial engrosada, presencia de fragmentos osteocondrales y reacción dolorosa durante la palpación articular y su movilización pasiva. La asignación de grados medios $(0,5)$ fue permitida.

Las medidas ecográficas fueron realizadas siempre en el mismo punto anatómico (recesos sinoviales dorsomedial y dorsolateral, inmediatamente por debajo de los maléolos medial y lateral) en cada caballo y por el mismo clínico, para esto se realizaron marcas en la piel con una maquinilla de afeitar. Las ecografías fueron realizadas con un equipo de ecografía Acuson-Aspen (UDS, Mountain View CA, EE.UU.) con una sonda lineal de $10 \mathrm{MHz}$.

Evaluación del grado de cojera. El grado de cojera fue calificado de 0-5, según los parámetros de la AAEP (1991). La asignación de grados medios $(0,5)$ fue permitida. Solamente se consideró el grado de cojera al trote en línea recta sobre una superficie dura. Los animales siempre fueron manejados por el mismo operario.

\section{DISEÑO DEL ESTUDIO}

Se realizó identificación aleatoria de los caballos con un número del 1-11. Se obtuvieron los datos bioquímicos de las concentraciones sinoviales basales de NO, PGE2 y HA. Paralelamente, se establecieron las concentraciones plasmáticas de HA (T0). Los pacientes fueron asignados al azar en dos grupos, tratamiento (n: 6) y control ( $n: 5)$. En ningún momento los investigadores pudieron conocer el grupo al que pertenecía cada animal. Esa información sólo fue revelada por la empresa dueña de la molécula al final del estudio. El grupo de caballos con tratamiento recibió $250 \mathrm{mg} /$ día de ácido hialurónico granulado, mezclado con celulosa microcristalizada. El grupo control recibió $250 \mathrm{mg} /$ día de celulosa microcristalizada (placebo). Los caballos recibieron el tratamiento asignado (HA oral o placebo) durante 60 días (T60). La evaluación del grado de efusión (distensión) sinovial, grado de cojera y las determinaciones bioquímicas fueron nuevamente realizadas al finalizar el tratamiento (60 días -T60-) y un mes después (T90). Las últimas determinaciones clínicas y bioquímicas fueron realizadas con el objetivo de conocer el efecto terapéutico residual de la sustancia evaluada (acción sintomática lenta en OCD).

\section{DETERMINACIONES BIOQUÍMICAS EN PLASMA} Y LÍQUIDO SINOVIAL

Las muestras de sangre entera fueron recolectadas de la vena yugular y depositadas en tubos de citrato de sodio al 3,8\% v/v con capacidad para $5 \mathrm{ml}$. Las muestras de sangre fueron transportadas en una nevera con hielo, hasta su procesamiento en el laboratorio. Cada articulación fue asépticamente preparada para punción articular y extracción de líquido sinovial, previa sedación con un bolo intravenoso 
de detomidina (40 $\mu \mathrm{g} / \mathrm{kg}$ ) (Domosedan, Pfizer, España) y tartrato de butorfanol $(0,02 \mathrm{mg} / \mathrm{kg}$ ) (Torbugesic, Fort Dodge Laboratories Inc, España). Un ml de líquido sinovial fue depositado en un tubo con EDTA para citología (recuento celular y determinación de proteína total). Otro $\mathrm{ml}$ fue depositado en un tubo con heparina de litio (determinación de NO, PGE2 y HA) e inmediatamente centrifugado a $1780 \times \mathrm{x}$ durante 5 minutos. Seguidamente se extrajo el líquido sinovial (aproximadamente $0,7 \mathrm{ml}$ ) libre de células. El sobrenadante fue depositado en tubos Ependorf y transportado de la misma manera que las muestras de sangre. Una vez en el laboratorio, la sangre citratada fue centrifugada a $1780 \mathrm{x}$ g durante 5 minutos, el plasma sobrenadante (determinación de HA) fue extraído y depositado en tubos Ependorf. Todas las muestras fueron congeladas a $-70{ }^{\circ} \mathrm{C}$, hasta su determinación bioquímica.

Determinación del óxido nítrico en el líquido sinovial. Se determinaron las concentraciones de NO en las muestras de líquido sinovial a través de su producto de degradación, los nitritos $\left(\mathrm{NO}_{2}\right)$, por medio del ensayo de diazotización (reacción de Greiss), previa reducción de los nitratos (Archer 1993). Se sabe que el $\mathrm{NO}_{2}$ es un producto final y estable del $\mathrm{NO}$ en una cantidad aproximada de $51,5 \pm 3,5 \%$ del total NO producido (von Rechemberg y col 2000). Primero se construyó una recta patrón a partir de un estándar de nitrito sódico al $100 \mathrm{mM}$. La recta fue iniciada en una dilución 1/1000 del estándar con una concentración máxima de $100 \mu \mathrm{M}$. Las muestras fueron centrifugadas a $1780 \mathrm{x} g$ durante 10 minutos y sin diluir fueron depositadas en alícuotas de $100 \mu \mathrm{l}$ en placas de 96 pozuelos (Ref. 35-3915, BD, New Jersey NJ, EE.UU.). El contenido de cada pozuelo fue mezclado con $100 \mu \mathrm{l}$ de una solución de trabajo compuesta por dos partes iguales de reactivo $\mathrm{A}$ ( $3 \mathrm{ml}$ de ácido fosfórico al 5\%, $51 \mathrm{ml}$ de agua y sulfanilamida al $1 \%(0,5 \mathrm{~g} / 50 \mathrm{ml}$ de ácido fosfórico al $5 \%)$ ) y reactivo B (N-1-Napthilethileno diamina (NEDA) al 0,1\% (0,05 g en $50 \mathrm{ml}$ de agua)).

Las muestras permanecieron incubadas a temperatura ambiente durante 10 minutos y luego fueron medidas en un lector de ELISA a un nivel de absorbancia de $540 \mathrm{~nm}$. A partir de la absorbancia se calculó la recta patrón y sobre la misma se estableció la concentración de nitritos de las muestras (Archer 1993). La sensibilidad del método fue de $1 \mu \mathrm{M}$. Todos los resultados se expresaron como $\mu \mathrm{M}$.

Determinación de la prostaglandina E2 en el líquido sinovial. Las concentraciones de PGE2 fueron determinadas mediante una prueba de ELISA en sándwich construida con anticuerpos comerciales (R\&D Systems, Abingdon, Reino Unido) para seres humanos. Se sabe que la estructura de la PGE2 está muy conservada entre especies y no existe reactividad cruzada de esta molécula con otros eicosanoides, por tal razón la prueba humana puede ser usada en el caballo (von Rechemberg y col 2000). El ensayo fue realizado de acuerdo a las instrucciones del fabricante. La lectura de absorbancia fue realizada a $405 \mathrm{~nm}$ mediante un lector de ELISA. La detección límite del ensayo fue de 15 pg/ml (von Rechemberg y col 2000).

Determinación del ácido hialurónico en plasma y líquido sinovial. El HA fue determinado mediante radioinmunoanálisis (RIA), con un kit comercial (HA Test, Pharmacia \& Upjohn Diagnostics AB, Uppsala, Suecia). La prueba se basó en la mezcla de $200 \mu$ l de proteínas de unión de ácido hialurónico aisladas de cartílago bovino (HABP-125) con $100 \mu \mathrm{l}$ de la muestra correspondiente de plasma o líquido sinovial. Las muestras fueron incubadas a $4-20^{\circ} \mathrm{C}$ durante 60 minutos, seguidamente se agregaron $100 \mu \mathrm{l}$ de AH-sefarosa y se reincubó a la misma temperatura durante 45 minutos adicionales. Luego se agregaron $2 \mathrm{ml}$ de solución de decantación y se centrifugó la muestra a $600 \times g$ durante 10 minutos. Las concentraciones de HA de las muestras fueron medidas mediante un contador gamma. El principio de la prueba consiste en cuantificar la cantidad de HABP-125 no unida al ácido hialurónico. De esta manera la radiactividad medida es inversamente proporcional a la cantidad de HA presente en la muestra (Guechot y col 1996).

\section{ANÁLISIS ESTADÍSTICO}

Los datos obtenidos fueron analizados con el programa estadístico SPSS versión 16.0 (SPSS Inc, Chicago IL, EE.UU.). Los valores de todas las variables evaluadas, a excepción de las concentraciones de proteína total del fluido sinovial, fueron analizados mediante pruebas no paramétricas, ya que estos datos presentaron una distribución no paramétrica (prueba de Shaphiro-Wilk $\mathrm{P}>0,05)$. Los grados de efusión sinovial y cojera y las concentraciones de NO, PGE2 y HA fueron analizados mediante un ANOVA no paramétrico de dos vías (ANOVA de Friedman). Se estableció que en el caso de hallar diferencias estadísticamente significativas se realizarían comparaciones pareadas no paramétricas de Wilcoxon (Dawson-Saunders y Trap 1993). Las concentraciones de proteína total fueron analizadas mediante un ANOVA de dos vías. Se estableció que en el caso de hallar diferencias estadísticamente significativas se realizarían comparaciones pareadas de Tuckey. De manera general se hizo un análisis de correlaciones entre las variables evaluadas. Un valor de $\mathrm{P}<0,05$ fue aceptado como significativo para todas las pruebas. Los resultados de las variables no paramétricas fueron presentados como medianas (rangos). Los datos de las variables paramétricas fueron presentados como medias (desviación estándar).

\section{RESULTADOS}

El estudio radiológico reveló que 10 caballos presentaban un fragmento osteocondral en la cresta intermedia de la tibia y uno presentaba un fragmento en el labio lateral de 
la tróclea del astrágalo. El grupo que recibió el tratamiento con HA estuvo conformado únicamente por caballos con fragmentación osteocondral de la cresta intermedia de la tibia. Los caballos incluidos presentaban efusión sinovial con un rango de calificación de 0,5-3 (mediana 1,5) (cuadro 1). Seis de los 11 caballos no presentaban cojera, los cinco restantes presentaban un grado de cojera que oscilaba en un rango de 0,5-2 grados. La mediana general del grado de cojera en los pacientes seleccionados fue de 0 (rango 0-2). En el cuadro 1 se presentan los registros del grado de efusión sinovial y cojera para cada uno de los grupos durante el tiempo.

El análisis citológico inicial del líquido sinovial de los 11 caballos incluidos presentó recuento celular mediano bajo (150 células $/ \mu \mathrm{L}$ (rango: $50-200$ células $/ \mu \mathrm{L}$ ), caracterizado por células mononucleares. Los caballos incluidos presentaron un nivel medio de proteínas totales en líquido sinovial de 1,2 $\pm-0,36 \mathrm{~g} / \mathrm{dL}$. Las concentraciones medianas de NO en líquido sinovial fueron de 2,92 $\mu \mathrm{M}$ (rango 1,33$6,27 \mu \mathrm{M})$. La PGE2 presentó niveles medianos en el líquido sinovial de 130,6 pg/mL (rango 41-231,7 pg/mL). El HA presentó concentraciones sinoviales medianas de $312,4 \mathrm{mg} / \mathrm{L}$ (rango 69,8-543,5 mg/L) y niveles plasmáticos medianos de 42,5 $\mu \mathrm{g} / \mathrm{L}$ (rango 21-109 $\mu \mathrm{g} / \mathrm{L}$ ). En el cuadro 2 se presentan los registros de los recuentos celulares y los metabolitos sinoviales y plasmáticos estudiados para cada uno de los grupos durante el tiempo.

Al finalizar el suministro del HA oral o del placebo a los 60 días del experimento (T60), ninguno de los caballos manifestó reacciones clínicas adversas. De manera general, no se observó ninguna diferencia estadísticamente significativa entre todos los parámetros clínicos (cuadro 1) y bioquímicos evaluados a los 60 y 90 días respectivamente (cuadro 2). Además, no se detectó ninguna correlación estadísticamente significativa entre los parámetros evaluados.
La impresión global a los 60 y 90 días postratamiento fue una tendencia hacia la mejoría clínica (disminución del grado de efusión sinovial (figura 1A) y del grado de cojera (cuadro 1) en los caballos que recibieron HA oral respecto al grupo placebo. Paradójicamente, los caballos que recibieron HA oral presentaron una tendencia a tener mayores concentraciones sinoviales de NO (cuadro 2 y figura 1B) y PGE2 (cuadro 2 y figura 1C) que el grupo control. Por otra parte, los caballos que recibieron HA oral tuvieron una tendencia a presentar menores concentraciones plasmáticas de HA (cuadro 2) y paralelamente concentraciones mayores de esa sustancia en el líquido sinovial (cuadro 2 y figura 1D), respecto al grupo control.

\section{DISCUSIÓN}

Según el conocimiento de los autores, este es el primer estudio doble ciego controlado en el que se evaluaron tanto parámetros clínicos como bioquímicos en caballos Andaluces jóvenes con OCD de la articulación tarsocrural, con el objetivo de conocer los efectos de un tratamiento oral con HA. Aunque en este estudio únicamente se observaron tendencias y no diferencias estadísticamente significativas entre los parámetros clínicos y bioquímicos evaluados, es necesario resaltar el valor científico de su diseño.

La mejoría clínica (pero no estadística) evidenciada en un menor valor mediano y en una disminución del rango del grado de efusión sinovial e incluso del grado de cojera de los caballos tratados con HA oral (cuadro 1 y figura 1) respecto al grupo placebo, podría coincidir con los resultados del trabajo de Bergin y col (2006), en el que caballos operados de OCD del tarso y que recibieron un suplemento oral de $100 \mathrm{mg}$ de HA durante 30 días presentaron disminución estadísticamente significativa del grado de efusión sinovial respecto al grupo placebo (Bergin y col 2006).

Cuadro 1. Mediana y rangos del grado de efusión articular y cojera en los caballos tratados con ácido hialurónico oral (T) y el grupo control (C).* group $(\mathrm{C}) *$

Median and ranges of the degree of lameness and synovial effusion of the oral hyaluronan horses' treated group (T) and the placebo

\begin{tabular}{lcccccc}
\hline \multirow{2}{*}{ Variable } & \multicolumn{2}{c}{$\mathrm{T} 0$} & \multicolumn{2}{c}{$\mathrm{T} 60$} & $\mathrm{~T} 90$ & $\mathrm{~T}$ \\
\cline { 2 - 7 } & $\mathrm{T}$ & $\mathrm{C}$ & $\mathrm{T}$ & $\mathrm{C}$ & $\mathrm{C}$ & 0,75 \\
Grado efusión sinovial & 1,25 & 2 & 1 & 2 & $(0,5-1,5)$ & $(0,5-3)$ \\
Grado de cojera & $(0,5-2,5)$ & $(0,5-3)$ & $(0,5-1,5)$ & $(0,5-3)$ & 0 & 1 \\
& 0 & 1,5 & 0 & 1 & $(0-0,5)$ & $(0-1,5)$ \\
\hline
\end{tabular}

T0 = Inicio del estudio. T60 $=60$ días del inicio del experimento. T90 = 90 días del inicio del experimento (fase final). $*=$ Todos los datos son presentados como medianas (rangos). No se presentaron diferencias estadísticamente significativas durante el tiempo para cada uno de los parámetros evaluados en cada grupo $(\mathrm{P}>0,05)$.

$\mathrm{T} 0=$ Begin of the study. T60 $=60$ days after beginning of the experiment. T90 $=90$ days after the beginning of the experiment (final phase).* $*$ All the data are presented as medians (ranges). No statistically significant differences were found for any of the parameters evaluated during the time for each group $(\mathrm{P}>0.05)$. 
Cuadro 2. Hallazgos citológicos sinoviales y concentración sinovial de proteína total, óxido nítrico, prostaglandina E2 y ácido hialurónico y concentración plasmática de ácido hialurónico en los caballos tratados con ácido hialurónico oral (T) y el grupo control (C).*

Synovial fluid cytological findings and synovial fluid concentration of total protein, nitric oxide, prostaglandin E2 and, hyaluronan and, plasmatic concentration of hyaluronan of the oral hyaluronan horses' treated group (T) and the placebo group (C).*

\begin{tabular}{|c|c|c|c|c|c|c|}
\hline \multirow{2}{*}{ Variable } & \multicolumn{2}{|c|}{ T0 } & \multicolumn{2}{|c|}{ T60 } & \multicolumn{2}{|c|}{ T90 } \\
\hline & $\mathrm{T}$ & $\mathrm{C}$ & $\mathrm{T}$ & $\mathrm{C}$ & $\mathrm{T}$ & $\mathrm{C}$ \\
\hline Células/ $\mu \mathrm{L}$ & $\begin{array}{c}190 \\
(50-200)\end{array}$ & $\begin{array}{c}180 \\
(60-200)\end{array}$ & $\begin{array}{c}190 \\
(70-210)\end{array}$ & $\begin{array}{c}200 \\
(90-250)\end{array}$ & $\begin{array}{c}170 \\
(80-230)\end{array}$ & $\begin{array}{c}190 \\
(80-230)\end{array}$ \\
\hline Proteína total sinovial $(\mathrm{g} / \mathrm{dL})^{* *}$ & $\begin{array}{c}1,33 \\
( \pm 0,36)\end{array}$ & $\begin{array}{c}1 \\
( \pm 0,32)\end{array}$ & $\begin{array}{c}1,06 \\
( \pm 0,31)\end{array}$ & $\begin{array}{c}0,91 \\
( \pm 0,13)\end{array}$ & $\begin{array}{c}0,92 \\
( \pm 0,16)\end{array}$ & $\begin{array}{c}0,78 \\
( \pm 0,6)\end{array}$ \\
\hline Óxido nítrico sinovial (NO2 $\mu \mathrm{M})$ & $\begin{array}{c}2,53 \\
(1,3-3,7)\end{array}$ & $\begin{array}{c}3,07 \\
(1,7-3,6)\end{array}$ & $\begin{array}{c}3,02 \\
(1,7-6,2)\end{array}$ & $\begin{array}{c}3,0 \\
(1,9-3,9)\end{array}$ & $\begin{array}{c}3,9 \\
(2,9-8,1)\end{array}$ & $\begin{array}{c}2,3 \\
(1,6-2,9)\end{array}$ \\
\hline Prostaglandina E2 sinovial $(\mathrm{pg} / \mathrm{mL})$ & $\begin{array}{c}139,8 \\
(88-232)\end{array}$ & $\begin{array}{c}130,6 \\
(41-181)\end{array}$ & $\begin{array}{c}78,2 \\
(52-136)\end{array}$ & $\begin{array}{c}71,6 \\
(56-96)\end{array}$ & $\begin{array}{c}97,6 \\
(50-324)\end{array}$ & $\begin{array}{c}68,3 \\
(29-77)\end{array}$ \\
\hline Ácido hialurónico sinovial (mg/L) & $\begin{array}{c}327,4 \\
(179-499)\end{array}$ & $\begin{array}{c}288,4 \\
(70-544)\end{array}$ & $\begin{array}{c}386,1 \\
(253-520)\end{array}$ & $\begin{array}{c}258,8 \\
(46-403)\end{array}$ & $\begin{array}{c}347,7 \\
(157-743)\end{array}$ & $\begin{array}{c}395,4 \\
(48-415)\end{array}$ \\
\hline Ácido hialurónico plasmático $(\mu \mathrm{g} / \mathrm{L})$ & $\begin{array}{c}43 \\
(39-74)\end{array}$ & $\begin{array}{c}36 \\
(21-109)\end{array}$ & $\begin{array}{c}39 \\
(21-119)\end{array}$ & $\begin{array}{c}70 \\
(22-114)\end{array}$ & $\begin{array}{c}26 \\
(186-64)\end{array}$ & $\begin{array}{c}34 \\
(15-106)\end{array}$ \\
\hline
\end{tabular}

Iniciales y símbolos como en el cuadro $1 . * *=$ Los datos son presentados como promedio (desviación estándar). No se presentaron diferencias estadísticamente significativas durante el tiempo para cada uno de los parámetros evaluados en cada grupo $(\mathrm{P}>0,05)$.

Letters and symbols as in table $1 . * *=$ Data are presented as mean (standard deviation). No statistically significant differences were found for any of the parameters evaluated during the time for each group $(\mathrm{P}>0.05)$.

A

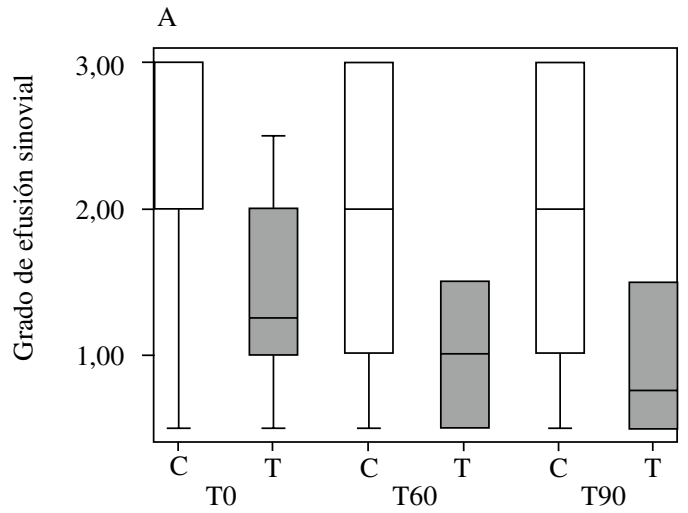

$\mathrm{C}$

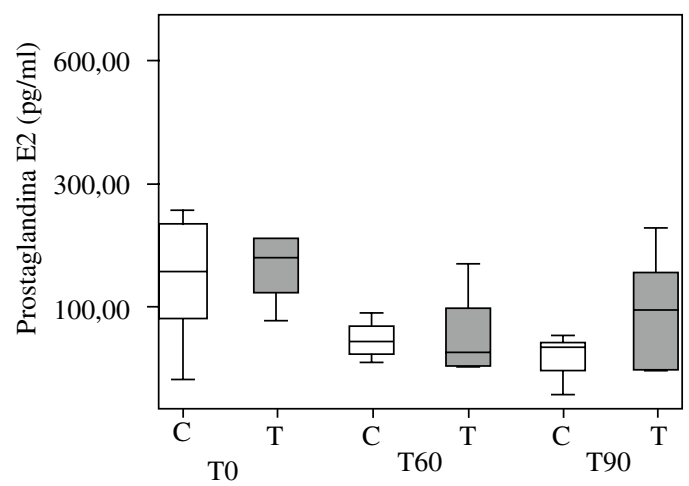

B

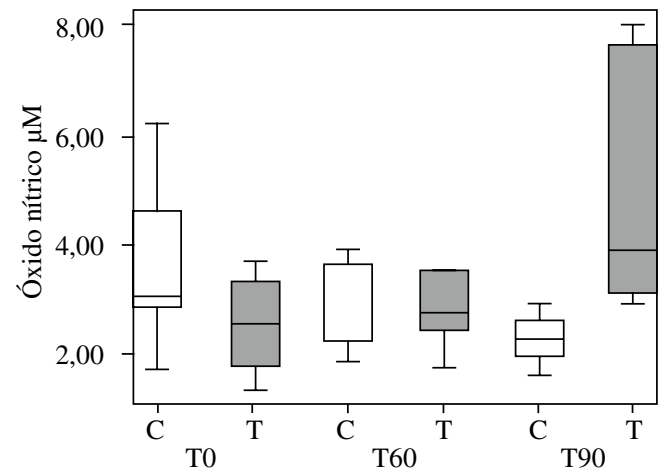

$\mathrm{D}$

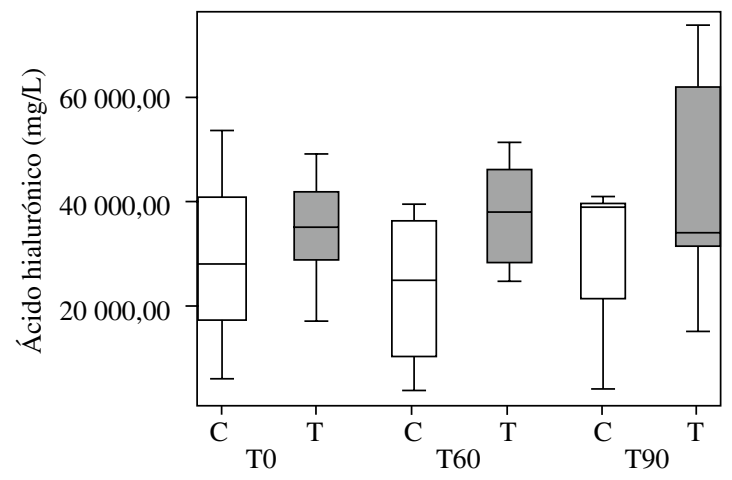

Figura 1. Gráficos de cajas y barras del grado de efusión sinovial (A) y concentraciones sinoviales de óxido nítrico (B), prostaglandina E2 (C) y ácido hialurónico (D). Demás letras como en el cuadro 1.

Chart boxes and bars of the degree of synovial effusion (A), and the nitric oxide (B), prostaglandin E2 (C) and, hyaluronic acid (D) synovial fluid levels. Other letters as in table 1. 
Los resultados obtenidos en la presente investigación produjeron dos interrogantes: 1) ¿Por qué el HA oral produjo una tendencia hacia el mejoramiento clínico en los caballos tratados, a pesar de que éstos presentaron mayores concentraciones de NO y PGE2 a los 60 y 90 días respecto al grupo control? 2) ¿Por qué los caballos tratados con HA oral presentaron tendencia a tener concentraciones plasmáticas inferiores de HA respecto al grupo control?

En primera instancia, estudios recientes en caballos jóvenes con OCD del tarso han encontrado a los eicosanoides (LTB4 y PGE2) (de Grauw y col 2006) y radicales libres (Gangl y col 2007), especialmente el NO, como protagonistas clave de la génesis y perpetuación de la efusión sinovial (cojera) y daño del cartílago articular (de Grauw y col 2006, Gangl y col 2007). Recientemente de Grauw y col (2006) documentaron concentraciones elevadas de PGE2 en el líquido sinovial de caballos con OCD del tarso $(362,3 \pm 93,6 \mathrm{pg} / \mathrm{ml})$ en comparación con el líquido sinovial de la misma articulación en caballos sanos $(127,3 \pm 33,6 \mathrm{pg} / \mathrm{ml})$. Cabe aclarar que las concentraciones medianas de PGE2 del fluido sinovial de los caballos del presente estudio (130,6 (rango 41$231,7) \mathrm{pg} / \mathrm{ml}$ ) fueron inferiores a las descritas por de Grauw y col (2006) para caballos con OCD del tarso, a pesar de que en ambas investigaciones se emplearon los anticuerpos de la misma casa comercial (R\&D Systems, EE.UU.). Sin embargo, en esta investigación el líquido sinovial fue depositado en tubos con heparina litio e inmediatamente centrifugados. En contraste de Grauw y col (2006) no usaron anticoagulante y la muestra permaneció durante una hora a temperatura ambiente antes de ser centrifugada. Es posible que las concentraciones de PGE2 reportadas por de Grauw y col (2006) hubiesen estado incrementadas frente a los posibles "valores reales" para la enfermedad (OCD), ya que ellos no depositaron el fluido sinovial en tubos con anticoagulante y tardaron (1 hora) en centrifugarlo. Es bien sabido que cuando los leucocitos entran en contacto con superficies extrañas (paredes del tubo) aumentan su actividad metabólica inflamatoria y además la heparina tiene la capacidad de prevenir ese efecto (Lappegård y col 2005). Así es posible que los valores de PGE2 de este trabajo sean más aproximados para caballos con OCD que los descritos por de Grauw y col (2006).

Los hallazgos del presente estudio están en contravía con los resultados reportados por Kawcak y col (1997), quienes encontraron que la aplicación intravenosa de HA disminuyó las concentraciones de PGE2 a los 72 días postoperatorios en caballos con OA experimental respecto al grupo control. Por otra parte, los resultados de la presente investigación podrían estar de acuerdo con los trabajos realizados por Tung y col $\left(2002^{\mathrm{a}, \mathrm{b}}\right)$, quienes encontraron que la adición de HA a condrocitos equinos condicionados con IL-1 no afectaba la expresión de iNOS o COX2, enzimas necesarias para la producción de $\mathrm{NO}$ o
PGE2 (Tung y col 2002a,b). Hallazgos parecidos en condrocitos humanos han sido más recientemente reportados por Maneiro y col (2004).

Es necesario tener en cuenta que varios investigadores consideran al NO (Schwentker y col 2002) y a la PGE2 (Gilroy y col 2004, Akaogi y col 2006, Stafford y Marnett 2008) como metabolitos reguladores y no promotores de la inflamación. Desde ese punto de vista se podría pensar que la tendencia a tener mayores concentraciones sinoviales de NO, PGE2 y HA, acompañadas de mejoría clínica evidente en el grupo de caballos que recibieron HA oral (respecto al grupo placebo), se pudo producir por una compleja interacción molecular y celular desencadenada por el fármaco evaluado. Sin embargo, esto es sólo una especulación que deberá ser resuelta mediante investigaciones más refinadas.

Por otra parte, se ha postulado que uno de los principales mecanismos de acción del HA en artropatías (degenerativas o traumáticas) es su efecto mecánico (viscosuplementación), el cual impide la interacción de las células mononucleares (inflamatorias) a través de sus receptores de superficie celular, tales como el CD44 (Howard y McIlwraith 1996). Es posible que la tendencia a tener mayores concentraciones sinoviales de HA en los caballos que recibieron HA oral respecto al grupo control hayan producido un efecto de recubrimiento celular de los macrófagos sinoviales y evitado así la interacción entre éstos, a pesar de las altas concentraciones de metabolitos proinflamatorios hallados (Howard y McIlwraith 1996).

El segundo interrogante planteado en esta discusión (¿por qué los caballos tratados con HA oral presentaron tendencia a tener concentraciones plasmáticas inferiores de HA respecto al grupo control?) podría ser explicado debido a que posiblemente el suplemento de HA oral haya producido un efecto de inducción enzimática (taquifilaxia) a nivel hepático con un consecuente aumento del metabolismo de degradación y depuración de esa sustancia, lo cual podría explicar la presencia de menores concentraciones de HA en plasma (Popot y col 2004); sin embargo, esto es únicamente una opinión especulativa. Otro hecho, es que la molécula evaluada de HA oral hubiera tenido un destino preferencial hacia las articulaciones. Aunque no fue presentado en la sección de los resultados, se pudo evidenciar un cierta tendencia a una correlación negativa entre las concentraciones plasmáticas y sinoviales de HA en los caballos del estudio. De manera general se observó que los caballos que tenían menores concentraciones plasmáticas de HA presentaban de manera paralela mayores concentraciones de HA sinovial. Sin embargo, esto no se pudo comprobar estadísticamente.

Finalmente, es posible pensar que el HA oral evaluado en este estudio podría ser considerado como una sustancia de acción sintomática lenta en la OCD, ya que sus efectos clínicos permanecieron durante más de un mes (T90) después de haber finalizado su administración (Howard y 
McIlwraith 1996), incluso uno de los caballos tratados, el cual presentaba una efusión sinovial grave (grado 3), mejoró ostensiblemente (grado 1) cuatro meses después de haber suspendido el tratamiento. El efecto de acción sintomática lenta de una sustancia determinada en las artropatías es una acción farmacológica sumamente importante para los nuevos componentes antiartríticos evaluados (tal como es el caso de la molécula investigada en este estudio). Muchas sustancias con acción sintomática en artropatías también pueden ser consideradas como fármacos moduladores de la respuesta biológica; es decir, aquellas sustancias que frenan el catabolismo articular y en algún caso promueven el anabolismo del cartílago articular (Howard y Mcllwraith 1996).

Se concluye que ninguna de las hipótesis planteadas en este estudio pudo ser corroborada estadísticamente. Al parecer el fármaco evaluado produjo una mejor tendencia en los resultados clínicos (disminución del grado de efusión sinovial) y en las concentraciones sinoviales de HA en los caballos tratados en comparación con lo observado en el grupo placebo. Según lo observado, se podría concluir que el mecanismo de acción del HA oral a dosis de $250 \mathrm{mg} /$ día, durante 60 días en caballos jóvenes con OCD, posiblemente no está relacionado con la disminución de la producción articular de NO y PGE2. Finalmente, el HA oral no produjo ningún efecto clínico adverso en los caballos tratados durante 60 días.

\section{RESUMEN}

El objetivo de este estudio fue evaluar los efectos clínicos y bioquímicos de una preparación oral de ácido hialurónico (HA) (Hyal-Joint ${ }^{\circledR}$ ) en caballos Andaluces jóvenes con osteocondrosis de la articulación tarsocrural. Las hipótesis planteadas fueron que la administración oral de HA es segura, disminuye el grado de efusión sinovial y las concentraciones sinoviales de óxido nítrico (NO) y prostaglandina E2 (PGE2) e incrementa los concentraciones plasmáticas y sinoviales de HA. Once caballos jóvenes pura raza español con OCD tarsocrural fueron enrolados en un estudio doble ciego controlado. 6 caballos recibieron $250 \mathrm{mg} /$ día de HA oral, durante 60 días, y 5 caballos recibieron un placebo. Se obtuvieron los valores iniciales del grado de efusión sinovial, NO, PGE2 y las concentraciones sinoviales y plasmáticas de HA. Se realizó análisis citológico con determinación de las concentraciones de proteína total. Los caballos fueron evaluados para los mismos parámetros al final del tratamiento (T60) y 30 días después (T90). No se encontraron diferencias estadísticamente significativas para los parámetros evaluados en el estudio en ambos grupos o durante el tiempo (comienzo, T60 y T90). Sin embargo, los pacientes tratados con HA oral presentaron tendencia a mostrar menor grado de efusión sinovial y mayores concentraciones de HA sinovial. El HA oral no produjo ningún efecto clínico adverso en los caballos tratados durante 60 días. A una dosis de $250 \mathrm{mg} / \mathrm{kg}$ el HA oral es seguro y bien tolerado en caballos.

\section{AGRADECIMIENTOS}

Los autores agradecen la gentil colaboración de todas las personas que participaron en la ejecución técnica del proyecto: Antoni Iborra, Laia Viñals, Paz Martínez, Ramón Deulofeu, Rafaela Cuenca y Pau Serra. También, agradecen el apoyo de la Compañía Bioibérica S.A, Barcelona, España, especialmente de Daniel Martínez y Ana Ubia.

\section{REFERENCIAS}

AAEP, American Association of Equine Practitioners. 1991. Guide for veterinary service and judging of equestrian events: definition and classification of lameness. Lexington, USA, Pp 19.

Akaogi J, T Nozaki, M Satoh, HYamada. 2006. Role of PGE2 and EP receptors in the pathogenesis of rheumatoid arthritis and as a novel therapeutic strategy. Endocr Metab Immune Disord Drug Targets 6, 383-394.

Archer S. 1993. Measurement of nitric oxide in biological models. FASEB $J$ 7, 340-360.

Balogh L, A Polyak, D Mathe, R Kiraly, J Thuroczy, M Terez, G Janoki, Y Ting, L R Bucci, AG Schauss. 2008. Absorption, uptake and tissue affinity of high-molecular-weight hyaluronan after oral administration in rats and dogs. J Agric Food Chem 56, 10582-10593.

Bergin BJ, SW Pierce, LR Bramlage, A Stromberg. 2006. Oral hyaluronan gel reduces post operative tarsocrural effusion in the yearling Thoroughbred. Equine Vet J 38, 375-378.

Carmona JU, CE Giraldo-Murillo. 2007. Fisiopatología y tratamiento convencional de la osteoartritis en el caballo. Vet Zootec 1, 60-73.

Dawson-Saunders B, RG Trapp. 1993. Bioestadística médica. $1^{\mathrm{a}}$ ed. Editorial Manual Moderno, México DF, México.

de Grauw JC, PA Brama, P Wiemer, H Brommer, $\mathrm{CH}$ van de Lest, PR van Weeren. 2006. Cartilage-derived biomarkers and lipid mediators of inflammation in horses with osteochondritis dissecans of the distal intermediate ridge of the tibia. Am J Vet Res 67, 1156-1162.

Frean SP, LA Abraham, P Lees. 1999. In vitro stimulation of equine articular cartilage proteoglycans synthesis by hyaluronan and carprofen. Res Vet Sci 67, 181-188.

Gangl M, D Serteyn, JP Lejeune, N Schneider, S Grulke, F Peters, T Vila, G Deby-Dupont, M Deberg, Y Henrotin. 2007. A type II-collagen derived peptide and its nitrated form as new markers of inflammation and cartilage degradation in equine osteochondral lesions. Res Vet Sci 82, 68-75.

Gilroy DW, J Newson, P Sawmynaden, DA Willoughby, JD Croxtall. 2004. A novel role for phospholipase $\mathrm{A}_{2}$ isoforms in the checkpoint control of acute inflammation. FASEB J 18, 489-498.

Guechot J, A Laudat, A Loria, L Serfaty, R Poupon, J Giboudeau. 1996. Diagnostic accuracy of hyaluronan and type III procollagen amino-terminal peptide serum assays as markers of liver fibrosis in chronic viral hepatitis C evaluated by ROC curve analysis. Clin Chem 42, 558-63.

Howard RD, CW McIlwraith. 1996. Hyaluronan and its use in the treatment of equine joint disease. In: Mcllwraith CW, Trotter GW (eds). Joint disease in the horse. WB Saunders Company, Philadelphia, USA, Pp 270-280.

Kawcak CE, DD Frisbie, GW Trotter, CW McIlwraith, SM Gillette, BE Powers, RMWalton. 1997. Effects of intravenous administration of sodium hyaluronate on carpal joints in exercising horses after arthroscopic surgery and osteochondral fragmentation. Am J Vet Res 58, 1132-1140.

Lappegård KT, J Riesenfeld, OL Brekke, G Bergseth, JD Lambris, TE Mollnes. 2005. Differential effect of heparin coating and complement inhibition on artificial surface-induced eicosanoid production. Ann Thorac Surg 79, 917-923.

Maneiro E, MC de Andrés, JL Fernández-Sueiro, F Galdo, FJ Blanco. 2004. The biological action of hyaluronan on human osteoartritic articular chondrocytes: the importance of molecular weight. Clin Exp Rheumatol 22, 307-312.

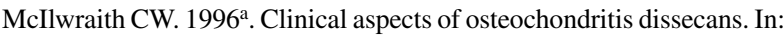
McIlwraith CW, Trotter GW (eds). Joint disease in the horse. WB Saunders Company, Philadelphia, USA, Pp 369-374.

Mcllwraith CW.1996 ${ }^{\mathrm{b}}$. General pathobiology of the joint and response to injury. In: McIlwraith $\mathrm{CW}$, Trotter $\mathrm{GW}$ (eds). Joint disease in the horse. WB Saunders Company, Philadelphia, USA, 40-70.

Olstad K, B Ytrehus, S Ekman, CS Carlson, NI Dolvik. 2007. Early lesions of osteochondrosis in the distal tibia of foals. J Orthop Res 25, 1094-105.

Palmer JL, AL Bertone.1994. Joint structure, biochemistry and biochemical disequilibrium in synovitis and equine joint disease. Equine Vet J 26, 263-277. 
Popot MA, J Bonnaire, J Guéchot, LP Toutain. 2004. Hyaluronan in horses: physiological production rate, plasma and synovial fluid concentrations in control conditions and following sodium hyaluronate administration. Equine Vet J 36, 482-487.

Robert C, JP Valette, JM Denoix. 2006. Correlation between routine radiographic findings and early racing career in French trotters. Equine Vet J Suppl 36, 473-488.

Schwentker A, Y Vodovotz, R Weller, TR Billiar. 2002. Nitric oxide and wound repair: role of cytokines? Nitric Oxide 7, 1-10.

Stafford JB, LJ Marnett. 2008. Prostaglandin E2 inhibits tumor necrosis factor-alpha RNA through PKA type I. Biochem Biophys Res Commun 366, 104-109.

Tung JT, PJ Venta, JP Caron. 2002a . Inducible oxide nitric expression in equine articular chondrocytes: effects of antiinflammatory compounds. Osteoarthritis Cartilage 10, 5-12.
Tung JT, PJ Venta, SW Eberhart, V Yuzbasiyan-Gurkan, L Alexander, JP

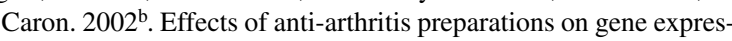
sion and enzyme activity of cyclooxygenase- 2 in cultured equine chondrocytes. Am J Vet Res 63, 1134-1139.

van Weeren PR. 2006. Etiology, diagnosis, and treatment of OC(D). Clin Tech Equine Pract 5, 248-258.

von Rechemberg B, CW McIlwraith, MK Akens, DD Frisbie, C Leutenegger, JA Auer. 2000. Spontaneous production of nitric oxide $(\mathrm{NO})$, prostaglandin $\left(\mathrm{PGE}_{2}\right)$, and neutral metalloproteinases (NMPs) in media of explant cultures of equine synovial membrane and articular cartilage from normal and osteoarthritic joints. Equine Vet J 32, 140-150.

Wittwer C, H Hamann, E Rosenberger, O Distl. 2006. Prevalence of osteochondrosis in the limb joints of South German Coldblood horses. J Vet Med A Physiol Pathol Clin Med 53, 531-539. 